\title{
Crown Size-Arch Space Relationships During Human Prenatal Dental Development
}

\author{
S. M. GARN, A. R. BURDI, W. J. BABLER, and R. ASP \\ Center for Human Growth and Development and The Department of Anatomy. The University of Michi- \\ gan School of Medicine, Ann Arbor, MI 48109 USA
}

As shown in composite reconstructions made from optically-projected measturements of $10 \mu$ frontal maxillofacial sections of 20 histologically normal fetuses and embryos in the $70.255 \mathrm{~mm} C R L$ range, individual differences in relative tooth size and interdental spacing are evident by 10.5-11 weeks of gestation, and age-corrected crown-size correlations $(r=0.40)$ approximate those in postnatal life. Despite an eight-fold increase in crown dimensions, interdental spacing remains approximately constant after $150 \mathrm{~mm} \mathrm{CRL}$, suggesting that the tooth organs or the periodontal membranes are able to maintain spacing, into the third trimester.

J Dent Res 58(2):554-559, February 1979

\section{Introduction.}

Although there are many studies on the development of tooth-spacing in the postnatal dentition, among them the classic contributions of Lundstrom ${ }^{1}$ and of Moorrees ${ }^{2}$, there is virtually no information on the prenatal origins of tooth-spacing or on the relationships between crown sizes and interdental spacing during odontogenesis. Yet it is possible that tooth-spacing is established early, and that crown-size relates to tooth spacing very early in prenatal development.

Again, there are a number of studies on the crown-size relationships of the deciduous dentition, including our own recent report ${ }^{3}$, but none (as far as we know) relating to crown size during the rapid dimensional growth of the tooth organs. Yet it is possible that individual differences in crown size are established early, and that intraindividual correlations in crown dimensions are established well before crown growth is completed.

Accordingly we have explored the early development of crown-size inter-relationships during embryonic and fetal development,

Received for publication December 19, 1977.

Accepted for publication June 19, 1978. and the interrelations between crown-size and spacing during prenatal time. To do this we have made use of unique embryological material, excluding spontaneous abortions and specimens showing any evidence of abnormality even in histologic examination. We have also combined one-dimensional measurements on serial stained sections into two-dimensional reconstructions so as to parallel measurements customarily made on casts of fully-emerged teeth.

\section{Materials and methods.}

This dimensional study is based upon two-dimensional reconstructions derived from serial $10 \mu$ frontal maxillo-facial sections of 20 grossly and histologically normal white embryos and fetuses between 70 and 255 $\mathrm{mm}$ in crown-rump length (CRL).

The specimens, uniformly obtained from elective (induced) abortions were fixed in $10 \%$ neutral buffered formalin, and the heads were frontally-sectioned at $10 \mu$. Successive frontal sections were projected in a Leitz projection microscope (Projektiskop) at a known magnification, carefully calibrated against a stage micrometer slide with $0.1 \mathrm{~mm}$ graduations (see Fig. 1). Measurements of the distance of the inner and outer surfaces of each tooth organ, relative to the septal or symphysial midline, were transferred to millimeter-squared graph paper, and the final measurements were made by vernier caliper directly on the $100 x$ two-dimensional composite. These techniques have been described in detail by Burdi $^{4}$ and Burdi and Lillie ${ }^{5}$, and are shown in Figure 2.

After spot-checking both Projektiskop and reconstruction measurements, and studies of intra-observer and inter-observer reliability, two sets of final measurements were entered into the Amdahl $760 \mathrm{~V} / 6 \mathrm{Com}-$ puter of the University of Michigan Com- 


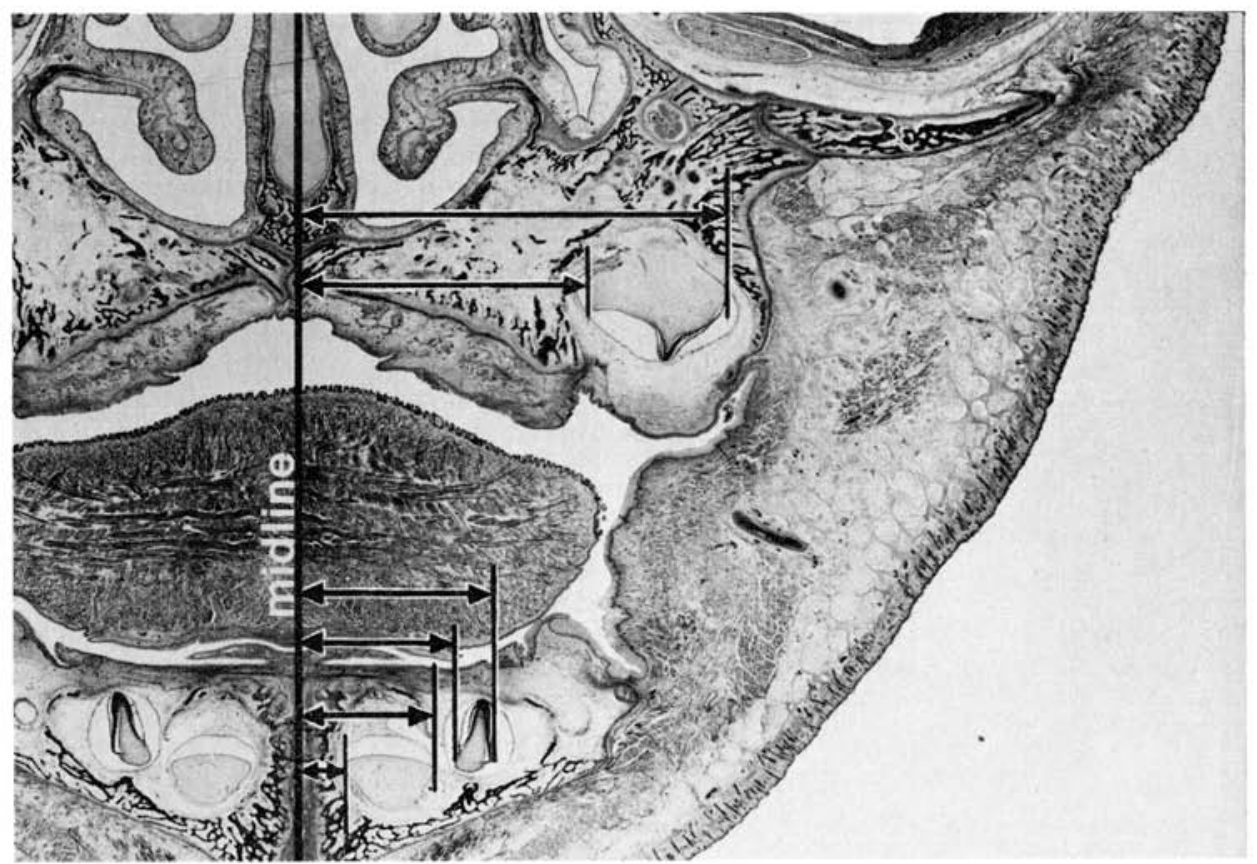

Fig. 1. $-10 \mu$ frontal section of a $225 \mathrm{~mm}$ CRL fetus showing the linear dimensions from the midline to the tooth organs as measured in optical projections and transferred to two-dimensional reconstructions. The lines indicate the distances measured from the midline to teeth on the right hemisection in each serial histologic section containing tooth material. Intra-observer replicability for these measurements is in excess of 0.996 .

puting Center (MTS). These included (a) mesiodistal (maximum) crown diameters of all deciduous crowns on the right hemisection and (b) corresponding interdental spaces. Total tooth material was also encoded as the sum of tooth diameters $(\Sigma T)$, and the corresponding interdental spaces $(\Sigma \mathrm{S})$ as the sum of interdental space beginning with the midline.

For certain of the analyses, individual crown-size diameters were expressed as residuals from the generalized regression lines, relative to crown-rump length (CRL). Crownto-crown correlations were age-corrected, so as to ascertain the magnitude of dimensional communality during the 10.5-28.0 week age range. The results, presented here, involved more than 12,000 individual distance measurements on over 3,000 histological sections of carefully selected embryos and fetuses.

\section{Results.}

In the first step of the computer-assisted data analysis, we explored the relationships between crown size and body size (expressed as CRL), and between interdental spacing and crown-rump length. Since each of the 10 deciduous teeth followed a family of curves relative to $\mathrm{CRL}$, as did the corresponding interdental spaces, developmental data on maxillary and mandibular incisors, canines and molars were combined as $(\Sigma \mathrm{T})$. Interdental spacing values $(\Sigma S)$ were similarly combined, on an individual basis.

As shown in the figure (Fig. 3), summed crown dimensions evidenced an eight-fold increase, in the $70-255 \mathrm{~mm} \mathrm{CRL}$ range. This increase with increasing age or crown-rump length was computer-fitted by the following equation, which accounts for 95 percent of variance.

$$
\begin{aligned}
& \Sigma \mathrm{T}=8.3 \times 10^{-6} \mathrm{CRL}^{3}+4.3 \times 10^{-2} \mathrm{CRL}^{2} \\
& -0.45 \mathrm{CRL}+18.4
\end{aligned}
$$

In contrast to the relatively simple sizeassociated increase in crown dimensions, there is a somewhat more complicated and slightly sigmoidal relationship between 


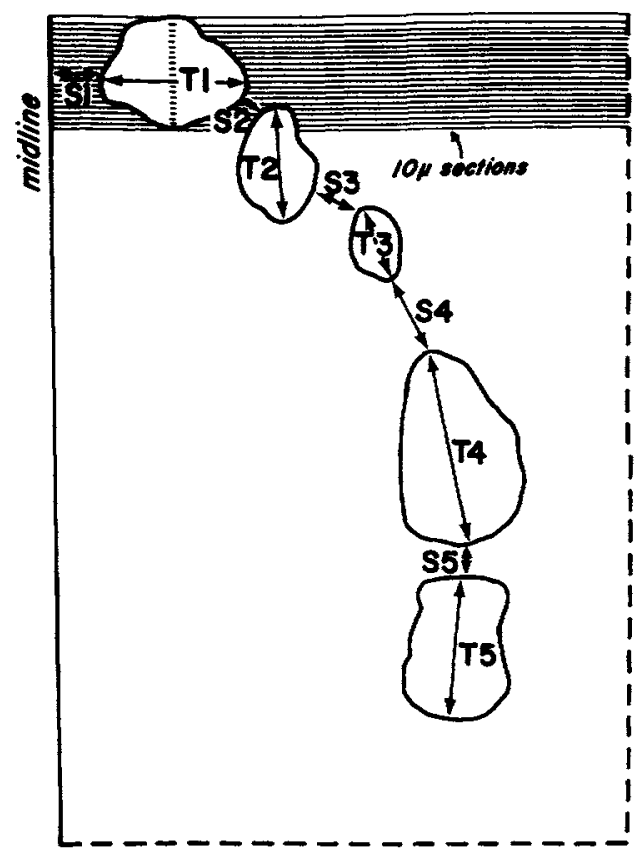

Fig. 2.-Two-dimensional reconstruction as made from serial "Projektiskop" measurements of histologic sections of a $155 \mathrm{~mm}$ fetus. Reproduced uniformly at $100 \mathrm{X}$, each serial section comprises $1 \mathrm{~mm}$ of space on the graphic reconstruction as shown here. Maximum crown diameters (T) and interdental spaces (S) are then measured with vernier calipers directly on the composite reconstruction. Inter-observer reliability in this stage of the work exceeds 0.97 .

crown-rump length (CRL) and interdental space $(\Sigma S)$. As shown in the figure, interdental space is approximately equal to crown size at 11 weeks (or $75 \mathrm{~mm} \mathrm{CRL)} \mathrm{but} \mathrm{then}$ decreases slightly. During most of the 17week period here considered, crown material is increasing in amount, while interdental space (after an initial decrease) remains relatively constant.

In the second step of the data analysis, we concentrated attention on intra-individual crown-size correlations involving 5 maxillary and 5 mandibular teeth as described above. Raw order correlations ( $\mathrm{r}_{0}$ ) were first computed using the Amdahl computer at the University of Michigan, and the resulting raw-order correlations were then corrected for crown-rump length by the use of partial correlations $\left(\mathrm{r}_{12.3}\right)$.

As shown in Table 1, mesiodistal crownsize dimensions are generally positively correlated in the $70-255 \mathrm{~mm}$ CRL range after correction for crown-rump length per se (19 out of 20 intercorrelations). These partial correlations furthermore are significant in 6 out of 20 cases, as against the one correlation that might be expected by chance. Overall, the mean partial correlation (derived from the mean $z$ transform of $r$ ) is 0.40 . It may further be noted that opponents show a higher correlation $(0.66)$ than do adjacent teeth $(r=0.52)$ and that magnitude of the partial correlations decreases with distance along the dental arch. Mean values of $r$ are highest for adjacent teeth, slightly lower for teeth separated by one intervening tooth $(\mathrm{r}=0.39)$, lower still for 2 intervening teeth $(\mathrm{r}=0.30)$, and lowest in 3 intervening teeth $(\mathrm{r}=0.22)$.

Stated more succinctly, age-corrected crown-size dimensions are significantly correlated within individual embryos and fetuses, indicating the existence of a prenatal size "field". Moreover, there is a distance "field" of decreasing relationship in crown size communalities in prenatal time, even before crown sizes are completed.

TABLE 1

INTRA-QUADRANT MESIODISTAL CROWN CORRELATIONS IN THE 70-255 MM FETUS

\begin{tabular}{|c|c|c|c|c|c|}
\hline \multirow[t]{2}{*}{ Tooth } & \multicolumn{5}{|c|}{ Tooth } \\
\hline & di1 & $\operatorname{di} 2$ & $\mathrm{dc}$ & $\mathrm{dm} 1$ & $\operatorname{dm} 2$ \\
\hline & \multicolumn{5}{|c|}{ maxillary teeth } \\
\hline dil & - & 0.40 & 0.40 & 0.13 & 0.39 \\
\hline $\mathrm{di2}$ & $0.61 *$ & - & 0.44 & 0.43 & $0.62 *$ \\
\hline $\mathrm{d} \dot{c}$ & 0.16 & $0.52 *$ & - & 0.34 & 0.40 \\
\hline $\operatorname{dm} 1$ & -0.03 & 0.19 & $0.64 *$ & - & 0.31 \\
\hline $\mathrm{dm} 2$ & 0.03 & 0.39 & $0.67 *$ & $0.76^{*}$ & - \\
\hline \multicolumn{6}{|c|}{ mandibular teeth } \\
\hline
\end{tabular}

*Significant at $p=.05$ or better. Mean $r$ approximates 0.40 . 
In the third step of the data analysis, we also investigated the relationships between crown size and interdental space, again on an age-correlated basis using partial correlations calculated with the Amdahl 470 V/6 computer. 25 partial correlations comparing crown sizes and interdental spaces were the subject of inquiry, pooling data from 50 intra-jaw correlations for economy of presentation.

As shown in Table 2, the mesiodistal crown-size values and the interdental space values are generally negatively correlated within quadrants $(22$ out of 25 pooled com- parisons, $\chi^{2}$ significant at $\mathrm{p}=.001$ ). Overall, the mean value of $r$ is -0.22 again pooling 50 intra-jaw crown-size vs. interdental space correlations. An additional 50 interjaw correlations, similarly pooled for simplicity of presentation, showed a similar and highly significant excess of negative correlations, and a very similar overall value of $r(-0.15)$.

It may be concluded, therefore, from these 100 age-corrected crown-size vs. interdental space correlations that crownsize and interdental space are slightly negatively correlated in prenatal time, after age-

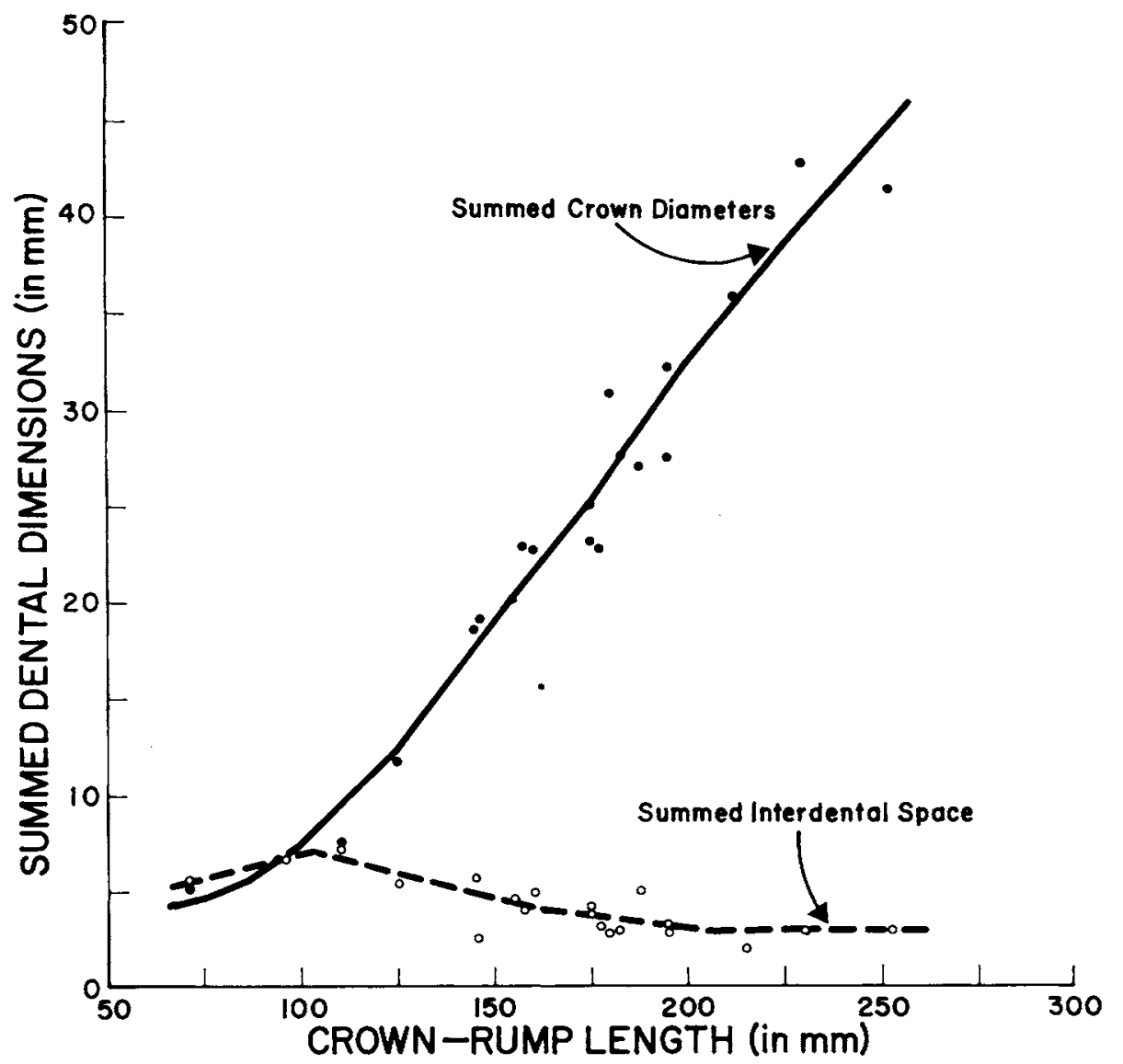

Fig. 3.- Comparative growth of summed crown diameters $(\Sigma T)$ and summed interdental space ( $\Sigma S)$ over the 10.5-28 week developmental span. As shown, summed crown diameters increase eightfold, while summed interdental space decreases slightly and then remains approximately constant during the remaining weeks. The fact that interdental space does not decrease despite the very large expansion of the tooth organs is indicative of space-maintaining mechanisms operating in the 155-225 $\mathrm{mm}$ size range. 
TABLE 2

CORRELATIONS BETWEEN MESIODISTAL CROWN DIMENSIONS AND INTERDENTAL SPACES DURING 10-28 WEEKS OF FETAL DEVELOPMENT

\begin{tabular}{llllll}
\hline Interdental Space & \multicolumn{5}{c}{ Tooth } \\
\cline { 2 - 6 } & \multicolumn{1}{c}{$\mathrm{di} 1$} & $\mathrm{di} 2$ & $\mathrm{dc}$ & $\mathrm{dm} 1$ & $\mathrm{dm} 2$ \\
\hline ML - di1 & $-0.75^{*}$ & -0.44 & -0.02 & -0.06 & -0.13 \\
$\mathrm{di1}-\mathrm{di} 2$ & -0.23 & -0.17 & -0.10 & -0.23 & +0.23 \\
$\mathrm{di} 2$ - C & -0.31 & -0.13 & -0.05 & -0.05 & -0.19 \\
$\mathrm{C}-\mathrm{dm} 1$ & $-0.48^{*}$ & $-0.56^{*}$ & $-0.52^{*}$ & +0.19 & -0.34 \\
$\mathrm{dm} 1-\mathrm{dm} 2$ & -0.06 & -0.26 & -0.14 & +0.06 & -0.23 \\
\hline
\end{tabular}

${ }^{*}$ Correlation significant at $p \leqslant 0.05$. Negative trend significant at $p \leqslant .001$ by sign test.

correction, but only to a small degree. This is to say that larger teeth are associated with slightly less space, both within quadrants and between the jaws. Crown size is therefore a factor in spacing, during odontogenesis, though not necessarily on a causal basis.

\section{Discussion.}

The findings in this developmental study provide much new information on the growth of deciduous tooth crowns and associated interdental spacing during the last two trimesters of prenatal life. Combining the onedimensional measurements of hemi-sections into two-dimensional reconstructions, it is now possible to duplicate analyses of crown size and spacing customarily accomplished on dental casts of children.

Even in the $70-255 \mathrm{~mm}$ crown-rump length range, tooth crown sizes prove to be moderately intercorrelated (after due correction for body size or gestation length). Interestingly, the magnitude of these prenatal crown-size inter-correlations ( $\mathrm{r} \cong 0.4$ ) is not markedly different from either deciduous or permanent tooth crown size intercorrelations as derived from plaster casts of Michigan children from the same general population. ${ }^{3,6}$ Clearly, some fetuses have large teeth overall, and others have small teeth, for their length of gestation, rather than the reciprocal relationships that would exist if large incisors (for example) were attained at the expense of the deciduous molars. Moreover, there is a size "field" of diminishing intensity in the prenatal dentition such that teeth more remote in the arch have less dimensional communality than teeth closer together in the dental arch. Again, these prenatal size fields resemble those demonstrable in the postnatal denti- tion, both deciduous and permanent. ${ }^{7,8}$

Plotting summed tooth sizes $(\Sigma T)$ against crown-rump length, there is a simple, nearlylinear relationship between them, with a working equation as given earlier. This makes it possible to identify large-toothed and small-toothed fetal specimens by their residuals against the trend line. It also offers the possibility of using summed crown sizes $(\Sigma \mathrm{T})$ as a new developmental reference standard for isolated fetuses or when other measures of gestation length are either lacking or impractical to attain.

As shown, the small but consistently negative correlations between summed crown size $(\Sigma \mathrm{T})$ and summed interdental space $(\Sigma \mathrm{S})$ suggest that larger crowns are associated with slightly less interdental space at every stage of early prenatal development. As with the individual differences in crown size, it is tempting to suggest that these reciprocal relationships may persist, on an individual basis, into the postnatal period. The notion of a reciprocal relationship between crown material and spacing is a classic notion in orthodontics, and it is intriguing to find some evidence in the early developmental period.

However, the fact that interdental space remains approximately constant during an eight-fold increase in crown-size is clear indication that "spacing" is far more than what is left in the arch after crown enlargement (otherwise "space" would quite disappear, in the course of crown expansion). Rather, there seem to be mechanisms that maintain space as the crowns enlarge. There is an analogy here in the mechanisms of spacing of such integumental structures as feathers ${ }^{9}$, hair ${ }^{10}$, and even the ridges and furrows in palmar dermatoglyphics. ${ }^{11}$ It is tempting to suggest that the developing periodontal 
membranes themselves serve as the spacemaintaining mechanisms, even as the tooth organs enlarge, thus encouraging compensatory growth for interdental space maintenance.

These analyses of the prenatal growth of crown material and concomitant changes in interdental spacing were made with postnatal models held in mind. They suggest that postnatal crown-size relationships do have prenatal origins, even though it is not feasible to follow embryos and fetuses in truly serial, longitudinal fashion. It is likely that intra-individual differences in crown size would be maintained from prenatal through postnatal time, and that crown-size relationships would also be maintained. It is further possible that postnatal differences in interdental spacing have their prenatal origins, as well.

Since a far larger independent series of grossly and histologically normal embryos and fetuses obtained from elective abortions may not be soon available, detailed confirmation of these findings may be slow to come. We have been especially careful, therefore, both in the data analysis and in generalizations therefrom. Certain of the findings so resemble the results of postnatal odontometric studies ${ }^{12}$ as to leave little doubt as to their validity. The major task as we see it is to elucidate the cellular mechanisms responsible for prenatal interdental space maintenance, because of the obvious relevance to problems of clinical importance.

\section{Acknowledgment.}

This investigation was supported by grants DE 03443 and DE 03610 from the National Institute of Dental Research. Seth Bidwell assisted in the manuscript completion.

\section{REFERENCES}

1. LUNDSTROM, A.: Aetiology of Crowding of the Teeth, Trans European Ortho Soc, p. 176, 1951.

2. MOORREES, C. F. A.: Dentition of the Growing Child, Harvard University Press, Cambridge, 1959.

3. GARN, S. M.; COLE, P.E.; and WAINRIGHT, R. L.: Dimensional Communalities of the Deciduous Teeth, J Dent Res 56:1208, 1977.

4. BURDI, A. R.: Morphogenesis of Mandibular Dental Arch Shape in Human Embryos, $J$ Dent Res 47:50-58, 1968.

5. BURDI, A. R.; and LILlIE, J. H.: A Catenary Analysis of the Maxillary Dental Arch during Human Embryogenesis, Anat Rec 154:13-20, 1966.

6. GARN, S.M.; COLE, P.E.; and WAINRIGHT, R. L.: Dimensional Correspondence Between Deciduous and Permanent Teeth, $J$ Dent Res $56: 1214,1977$.

7. GARN, S. M.: A Canine "Field" in the Sexual Dimorphism in Tooth Size, Nature 24: 1501-1502, 1966.

8. GARN, S. M.; LEWIS, A. V.; and KEREWSKY, R. S.; Size Interrelationships of the Mesial and Distal Teeth, $J$ Dent Res 44:350$354,1965$.

9. HOLMES, A.: The Pattern and Symmetry of the Adult Plumage Units in Relation to the Order and Locus of Origin of the Embryonic Feather Papillae, Amer $J$ Anat 56:513537,1935 .

10. RAWLES, M. E.: Skin and its Derivatives, in Analysis of Development, WILLIAR, B. H.; WEISS, P. A.; and HAMBURGER, V. (eds), W. B. Saunders Company, Philadelphia, 1955, pp. 499-519.

11. CUMMINS, H.: Epidermal Ridge Configurations in Developmental Defects, with Particular Reference to the Ontogenetic Factors which Condition Ridge Direction, Amer $J$ Anat 38:89 151, 1926.

12. MOORREES, C. F. A.; and REED, R. B.: Biometrics of Crowding and Spacing of the Teeth in the Mandible, Amer J Phys Anthrop $12: 77-88,1954$. 